\title{
Challenges of the WEB Device for Intracranial Aneurysms: How to Widen the Spectrum and Compare Favorably
}

$\mathrm{T}$ he authors undertook a prospective, multicenter study investigating the feasibility and safety of a new intra-aneurysmal flow disruptor, the WEB device (Sequent Medical, Aliso Viejo, California) in a cohort of 63 aneurysms. Two different models (WB DL and SL/SLS) were used, showing low overall morbidity and no mortality, with potential superiority of the newer SL/SLS model.

The authors must be commended for a thorough investigation of this important device, in which sound and comprehensive data acquisition during the initial safety and feasibility phase can facilitate detailed and longer follow-up studies, which are much needed for future efficacy interpretation.

The WEB device was primarily developed for treatment of wide-neck aneurysms, which may not be amenable to standalone coiling or even stent placement plus coiling. The authors convincingly demonstrated the feasibility of this new technique in a large subgroup of both ruptured and unruptured aneurysms, potentially widening the indication for endovascular treatment. To better understand the applicability of this new approach, documentation of the degree of immediate occlusion (even with adjunctive treatments) is essential; unfortunately, these results were not included in the current study. Although a control angiogram was obtained after device placement to determine flow stagnation (or filling adjacent to the deployed device), only the technical success rate (deployment of the WEB device in the target aneurysm) was provided. While analysis of technical feasibility is a prerequisite for any new technique to be investigated, analysis of success/efficacy will be the invariable next step to better determine the value of intraaneurysmal flow disruption. The authors will continue data collection up to 24 months after treatment, and we will be eagerly awaiting any short-term follow-up results on aneurysm occlusion to compare with their recent retrospective analysis with an aneurysm occlusion rate of $56.9 \% .{ }^{1}$ We are hopeful that data acquisition will be continued even beyond this point because newer data imply increasing relevance of particularly long-term durability up to 10 years.

A major clinical drawback for more complex endovascular treatment with stent or flow-diverter placement-apart from a potential increase in the operative complication rate-is the frequent necessity for (prolonged and/or double) antiplatelet treatment. Counteracting the thrombogenic effect of additional intraluminal devices can significantly increase the risk profile of any subsequent open surgical procedure, if not prohibiting it all altogether, a clinical dilemma not infrequently encountered, particularly in patients with ruptured aneurysms requiring temporary or permanent CSF diversion. If the WEB device can indeed facilitate the treatment of more wide-neck aneurysms with fewer parent artery constructs, it will be crucial to see additional data on the actual need for antiplatelet treatment with the WEB device.

In this study, the incidence of thromboembolic events was found to be in the range of $9 \%-23 \%$, depending on the type of device used and diagnosed by MR imaging and/or DSA. Routine MR imaging within 24 hours after the procedure in all patients may clarify the exact rate and type of this particular complication, also in the context of a particular antiplatelet regimen. If a much-needed decrease in these events can be achieved only with an intensification of antiplatelet treatment, the advantage of this new device may, in part, be limited to the ascribed ease of use and application. In this regard, the discrepancy in complication rates between the devices used (WEB DL versus SL/SLS) will also warrant further attention, and the effect of user experience/efficacy and learning curve will have to be separated from a device-related risk profile (SL/SLS with more rapid contrast stasis and a balanced radial force with conformability).

Reduction of periprocedural complications-independent from an individual learning curve-and an increase in efficacy are the basis for offering any new technique to future patients. This holds true in view of a potentially benign natural course with smaller, unruptured aneurysms, in which treatment recommendation must be based on the premise of a particularly low interventional risk profile with convincing long-term durability. Also, new and convincing evidence from large-volume, high-competence centers now suggests clinical equipoise for surgical treatment of anterior circulation aneurysms with 
longer follow-up, ${ }^{2}$ supported by other centers and data registries. ${ }^{3,4}$ With this compelling new technique of intra-aneurysmal flow disruption proficiently introduced by the authors, both the safety and long-term durability will have to compare favorably with what, for many, is currently considered the gold standard of treatment for wide-neck (MCA) aneurysms: surgical clipping.

\section{REFERENCES}

1. Papagiannaki C, Spelle L, Januel AC, et al. WEB intrasaccular flow disruptor-prospective, multicenter experience in 83 patients with 85 aneurysms. AJNR Am J Neuroradiol 2014;35:2106-11

2. Spetzler RF, McDougall C, Zabramski J, et al. The Barrow Ruptured Aneurysm Trial: 6-year results. In: Proceedings of the 82nd Annual
Scientific Meeting of the American Association of Neurological Surgeons, San Francisco, California. April 5-9, 2014

3. Bakker NA, Metzemaekers JD, Groen RJ, et al. International Subarachnoid Aneurysm Trial 2009: endovascular coiling of ruptured intracranial aneurysms has no significant advantage over neurosurgical clipping. Neurosurgery 2010;66:961-62

4. Gonda DD, Khalessi AA, McCutcheon BA, et al. Long-term follow-up of unruptured intracranial aneurysms repaired in California. J Neurosurg 2014;120:1349-57

G.A. Schubert

Department of Neurosurgery Cerebrovascular Section RWTH Aachen University Aachen, Germany

http://dx.doi.org/10.3174/ajnr.A4206 